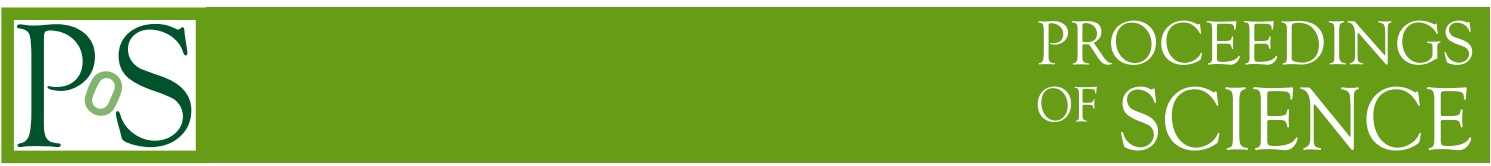

\title{
The T2K ND280 Upgrade
}

\section{Davide Sgalaberna on behalf of the T2K Collaboration ${ }^{a, *}$ \\ ${ }^{a}$ ETH Zurich, Institute for Particle Physics and Astrophysics, CH-8093 Zurich, Switzerland}

E-mail: davide.sgalaberna@cern.ch

In view of the J-PARC program of upgrades of the beam intensity, the $\mathrm{T} 2 \mathrm{~K}$ collaboration is preparing towards an increase of the exposure aimed at reaching sensitivity for leptonic $\mathrm{CP}$ violation at $3 \sigma$ level for a significant fraction of the possible $\delta_{C P}$ values. To reach this goal, an upgrade of the T2K near detector ND280 will be installed at J-PARC in 2022, with the aim of reducing the combined statistical and systematic uncertainties to better than $4 \%$. We have developed an innovative concept for this neutrino detection system, comprising the SuperFine-Grained-Detector (SuperFGD), two High Angle TPC (HA-TPC) and six TOF planes. The SuperFGD, a highly segmented scintillator detector, acting as a fully active target for the neutrino interactions, is a novel device with dimensions of approximately $1.9 \times 1.9 \times 0.6 \mathrm{~m}^{3}$ and a total mass of about 2 ton. It consists of about 2 millions small scintillator cubes each $1 \mathrm{~cm}^{3}$. Each cube is optically isolated. The signal readout from each cube is provided by wavelength shifting fibers inserted through the cubes and connected to micro-pixel avalanche photodiodes MPPCs. The total number of channels will be $\sim 60,000$. We have demonstrated that, by providing three $2 \mathrm{D}$ projections, this detector delivers excellent PID, timing, and tracking performance, including a $4 \pi$ angular acceptance, especially important for short proton and pion tracks. The HA-TPC will be used for $3 \mathrm{D}$ track reconstruction, momentum measurement and particle identification. These TPCs, with overall dimensions of $2 \times 2 \times 0.8 \mathrm{~m}^{3}$, will be equipped with 32 resistive Micromegas. The thin field cage ( $3 \mathrm{~cm}$ thickness, $4 \%$ rad. length) will be realized with laminated panels of Aramid and honeycomb covered with a kapton foil with copper strips. The $34 \times 42 \mathrm{~cm}^{2}$ resistive bulk Micromegas will use a $500 \mathrm{kOhm} / \mathrm{square}$ DLC foil to spread the charge over the pad plane, each pad being appr. $1 \mathrm{~cm}^{2}$. The front-end cards, based on the AFTER chip, will be mounted on the back of the Micromegas and parallel to its plane. The time-of-flight (TOF) detector will allow to reject events generated in the passive areas of the detector and improve particle identification. The TOF will consist of 6 planes with about $5 \mathrm{~m}^{2}$ surface area surrounding the SuperFGD and the TPCs. Each plane will be assembled with $2.2 \mathrm{~m}$ long cast plastic scintillator bars with light collected by arrays of large-area MPPCs from two ends. The time resolution at the bar centre is 150 ps. A report on the design of these detectors, their performance, the results of the test beam and the plan for the construction is provided.

40th International Conference on High Energy physics - ICHEP2020

July 28 - August 6, 2020

Prague, Czech Republic (virtual meeting)

\footnotetext{
${ }^{*}$ Speaker
} 


\section{Introduction}

T2K is a neutrino long-baseline experiment that measures the neutrino oscillations [1]. An intense (anti)neutrino beam peaked at $600 \mathrm{MeV}$ energy is produced at the J-PARC proton accelerator centre in Japan and is analyzed by two detectors: a magnetised near detector (ND280), placed 280 $\mathrm{m}$ away from the neutrino source, that measures the (anti)neutrino rate and spectrum unaffected by oscillations and a far detector (Super-Kamiokande), $295 \mathrm{~km}$ away, where the oscillation probability is near maximal. The measurement of $v_{\mu} \rightarrow v_{e}$ appearance with a non-zero $\theta_{13}$ [2] has opened the possibility to look for leptonic $\mathrm{CP}$ violation in neutrino oscillations: recently $\mathrm{T} 2 \mathrm{~K}$ has observed a first hint of maximal $\mathrm{CP}$ violation [3, 4]. The T2K collaboration is planning to extend the data taking program in order to collect at least $10 \times 10^{21}$ POT (protons on target) until before the start of Hyper-Kamiokande with the goal of searching for the first evidence of CP violation in the leptonic sector [5]. As shown in fig. 1 the phase II of the T2K experiment will be accompanied by a gradual increase of the neutrino beam intensity, up to about 1.3 MW at the end of the experiment. However in order to improve the sensitivity to search for $\mathrm{CP}$ violation, also a reduction of the systematic uncertainties is also required. For this reason the T2K collaboration has planned an upgrade of the near detector.

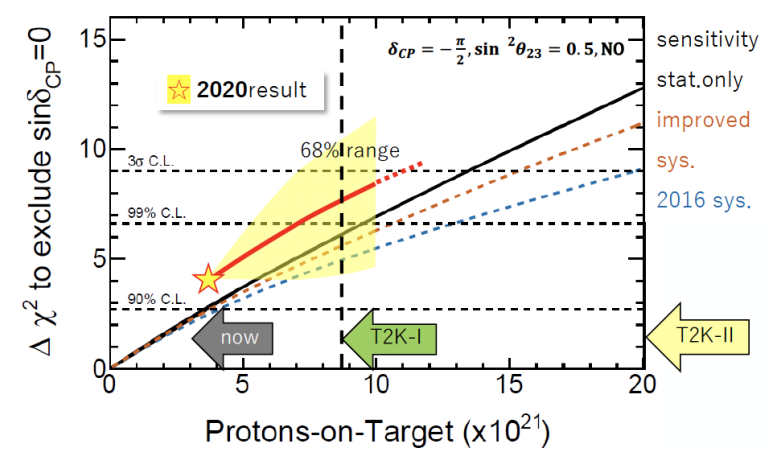

Figure 1: Expected beam power and POT accumulation as a function of the Japanese Fiscal Year (JFY) [5].

The ND280 detector is a magnetized detector composed by a $\pi^{0}$ detector (P0D) and two finegrained detectors (FGDs), acting as neutrino target, sandwiched between three Time Projection Chambers (TPCs). All the detectors are embedded inside an electromagnetic calorimeter (ECal). ND280 is optimized to detect particles propagating in the forward direction but not backward or perpendicular to the neutrino beam, where the muon detection efficiency is lower than $20 \%$ and is affected by large detector systematic uncertainties. For this reason, the T2K collaboration is planning to upgrade ND280 in order to improve its acceptance. The new configuration, described in [6], consists of a novel tracker, 2-ton horizontal plastic scintillator target, called Super Fine-Grained Detector (SuperFGD), with a size of 1.9 (width) $\times 0.6$ (height $) \times 1.9$ (length) $\mathrm{m}^{3}$, sandwiched between two horizontal TPCs, surrounded by a Time-of-Flight (ToF) detector, as shown in fig. 2. The new configuration will increase the total neutrino target mass up to about 4 tons with a $4 \pi$ acceptance. 


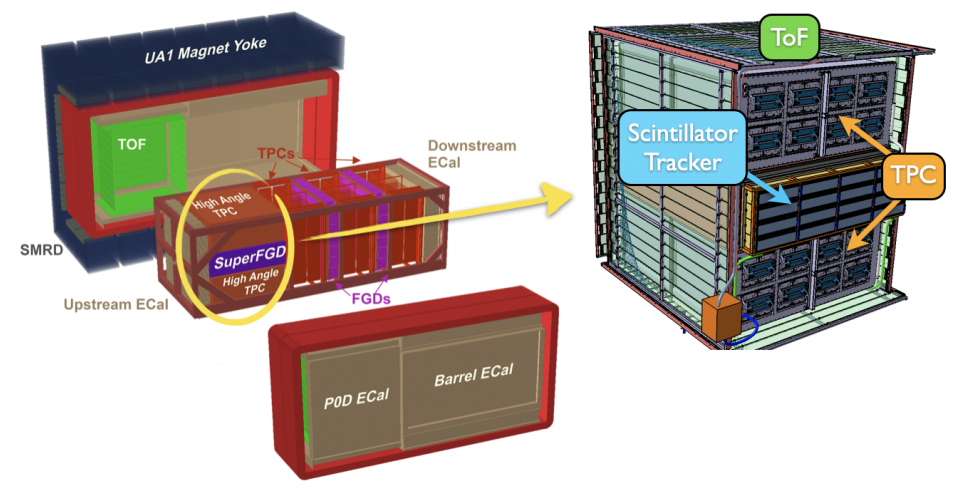

Figure 2: Picture of the upgraded ND280 configuration.

\section{The detectors}

The SuperFGD detector [7] consists of a matrix of approximately 2,000,000 optically-isolated cubes, each one $1 \mathrm{~cm}^{3}$. The total mass is about 2 tons to serve as target for neutrino interactions. The scintillation light produced by charged particles in the cubes is captured by three orthogonal wavelength shifting (WLS) fibers, each one read out by a Hamamatsu MultiPixel PhotoCounter (MPPC) and front-end electronics based on the one developed for the Baby MIND detector [8]. The concept of SuperFGD is described in [7]. Thanks to its high light yield, $\sim 40$ photoelectrons for a minimum ionizing particle (MIP) after $1 \mathrm{~m}$ attenuation in the WLS fiber, and its fine granularity, SuperFGD can provide a very good $\mathrm{dE} / \mathrm{dx}$ resolution and an excellent time resolution, about $0.9 \mathrm{~ns}$ per channel for a MIP. Moreover, thanks to its fine granularity, SuperFGD can precisely track particles at any angle and detect protons with a momentum threshold down to approximately $300 \mathrm{MeV} / \mathrm{c}$. The several beam tests at CERN allowed for the characterization of the response by exposing a SuperFGD prototype to a test beam of charged particles and measured its performance [9]. New data were also collected at LANL in Los Alamos by exposing two SuperFGD prototypes to a neutron test beam. Data analysis is ongoing. In Fig. 3, the concept of the SuperFGD detector and its performance for particle tracking and $\mathrm{dE} / \mathrm{dx}$ measurement is shown.
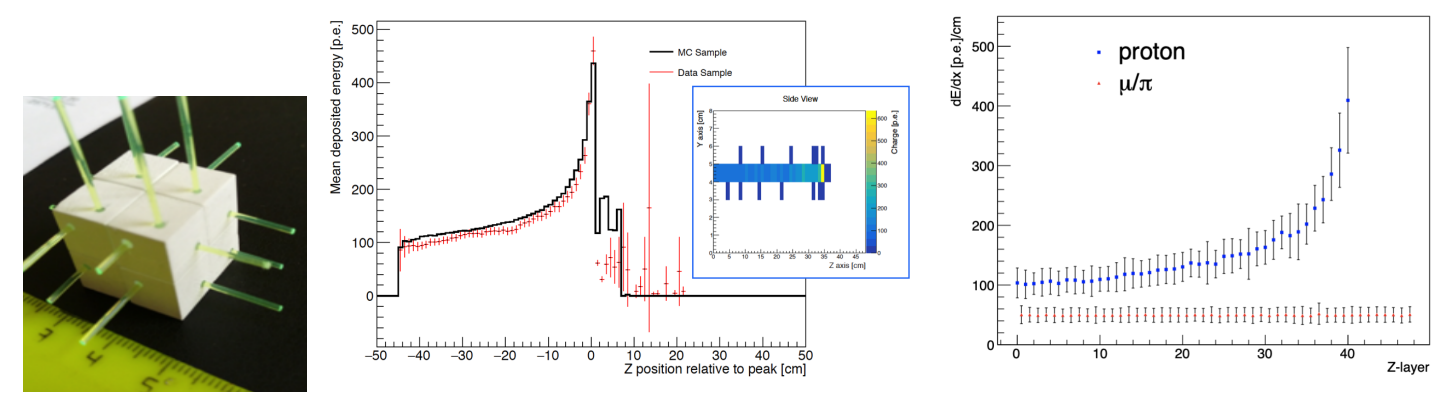

Figure 3: Left: picture of a small prototype of the Super-FGD. Center: measured Bragg-peak distribution from $800 \mathrm{MeV} / \mathrm{c}$ protons at CERN beam test, compared with simulations. Right: measured dE/dx for 800 $\mathrm{MeV} / \mathrm{c}$ protons and minimum-ionising particles (MIP) at the CERN beam test. 

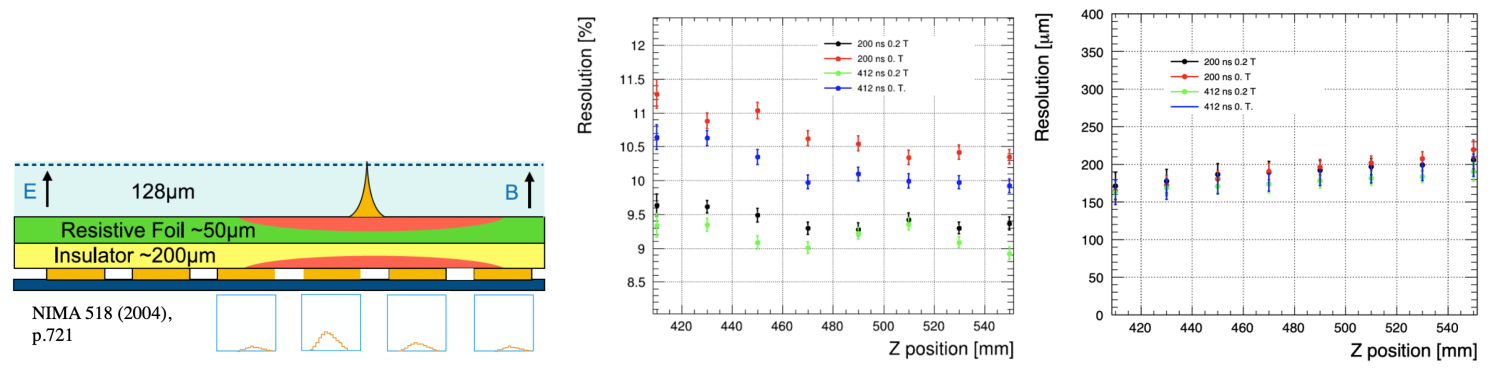

Figure 4: Left: concept of the resistive micromegas. Center: dE/dx resolution measured at DESY beam tests $(10 \mathrm{~cm}$ drift length) Right: space-point resolution measured at DESY beam tests.

The new TPCs will use an argon-based gas (Ar:CF4:iC4H10 - 95:3:2) and a new resistive micro-megas detector, shown in fig. 4, to protect from intrinsic spark and provide a better spatial resolution $[11,12]$. The drifted-electron signal will be spread over more sensitive pads (on average 2 or 3), providing a better space-point resolution. A thin rectangular field cage, with a drift length of $\sim 90 \mathrm{~cm}$ and only a few \% radiation length thickness, will provide a very uniform electric field along the transversal direction, parallel to the magnetic field. The minimum requirement for the new TPCs is to have the same performance of the TPCs already installed in ND280, i.e. $\mathrm{dE} / \mathrm{dx}$ resolution of $10 \%$ for MIP and $9 \%$ momentum resolution at $1 \mathrm{GeV} / \mathrm{c}$ with a space point resolution of $800 \mu \mathrm{m}$ with 64 space points. Beam tests were performed at CERN [13] and DESY. It was found that a dE/dx resolution of about $9 \%$ is achieved for $800 \mathrm{MeV} / \mathrm{c}$ positrons and the space-point resolution is improved by more than a factor three, i.e. better than $200 \mu \mathrm{m}$ with a drift distance of about $10 \mathrm{~cm}$ and a pad size of $1.01 \times 1.12 \mathrm{~cm}^{2}$.

One of the main issues in the track reconstruction is given by the determination of the track flip when timing is not precisely measured. In order to provide a precise timing, six ToF panels, made of plastic scintillator bars, will cover the new detectors. It can also be used to improve the PID when the $\mathrm{dE} / \mathrm{dx}$ measurement with the TPCs cannot provide enough separation. This is the case of positrons, whose $\mathrm{dE} / \mathrm{dx}$ is similar to the one of protons at $\sim 1 \mathrm{GeV} / \mathrm{c}$, or muons with a momentum of $\sim 0.2 \mathrm{GeV} / \mathrm{c}$. The detector consists of 12 (width) $\times 1$ (thickness) $\times 230$ (length) $\mathrm{cm}^{3}$ EJ-200 cast plastic scintillator bars, with an attenuation length of about $4 \mathrm{~m}$ [14]. The scintillation light propagates through the scintillator, without using any WLS fiber, and is read out at both ends with an array of eight $6 \times 6 \mathrm{~mm}^{2}$ MPPCs (S13360-6050PE). The measured time resolution is better than 150 ps (see fig. 5).

\section{Expected performance of the upgraded ND280}

The expected total number of selected CC- $v_{\mu}$ events will be doubled thanks to the increased neutrino target mass. As shown in fig. 6, the charged-current (CC) $v_{\mu}$ selection efficiency will be dramatically improved when muons are emitted at high angles or with backward direction. Thanks to the improved performance we expect to reduce the flux and cross-section systematic uncertainties by $30-40 \%$ with respect to the current ND280 configuration [6]. The background generated outside the fiducial volume will be reduced to below $1 \%$. Thanks to the detection of stopping muons with 

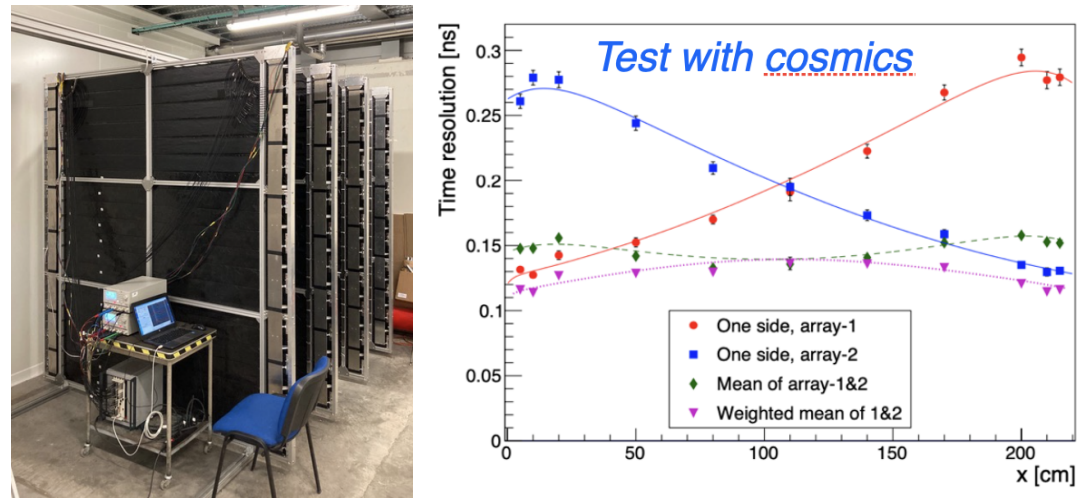

Figure 5: Left: ToF panels assembled at CERN. Right: ToF time resolution as a function of the interaction in the scintillator bars. A uniform resolution better than $150 \mathrm{ps}$ (pink) is achieved.

SuperFGD, an increase of $15-20 \%$ in the efficiency is expected with an average momentum-by-range resolution better than $3 \%$.

The improved detection of low-energy hadrons with full angular acceptance in SuperFGD will allow to perform detailed measurements in the projection transverse to the neutrino direction and probe nuclear effects [15]. At the same time other variables, like initial nucleon momentum and calorimetric energy can be studied. Based on MC, ND280 upgrade will have a better sensitivity to distinguish nuclear effects from flux normalization and will allow to constrain 2 particle - 2 hole processes at 2-3\% level and binding energy to $\sim 1 \mathrm{MeV}$ [6]. Moreover, SuperFGD is optimal to detect neutrons produced in neutrino interactions and possibly measure their time-of-flight, i.e. the energy. Simulations of neutrinos detected in SuperFGD showed that a detection efficiency ranging from 50\% to $90 \%$, depending on the neutron energy and direction, can be achieved with an energy resolution of about $20 \%$ for $100 \mathrm{MeV}$ neutrons. Moreover, it could be possible to select a neutron sample enhanced by antineutrino charged-current interactions in hydrogen with a proper cut on the transverse momentum imbalance [16].

\section{Conclusions}

In view of the T2K phase-II, the collaboration is upgrading the ND280 detector complex to further reduce the systematic uncertainties in the measurement of neutrino oscillations and improve the sensitivity to observing a violation of the CP symmetry in the leptonic sector. The project has been approved by the CERN SPSC and J-PARC PAC and it aims to start collecting first data in 2022. The upgrade of ND280 will enhance the T2K physics program in addition to the beamline upgrade in 2022 that will provide higher beam power from 0.5 up to $1.3 \mathrm{MW}$ before the start of Hyper-Kamiokande.

\section{References}

[1] K. Abe et. al (T2K Collaboration), Nuclear Instruments and Methods in Physics Research A 659, 106-135 (2011), 10.1016/j.nima.2011.06.067 


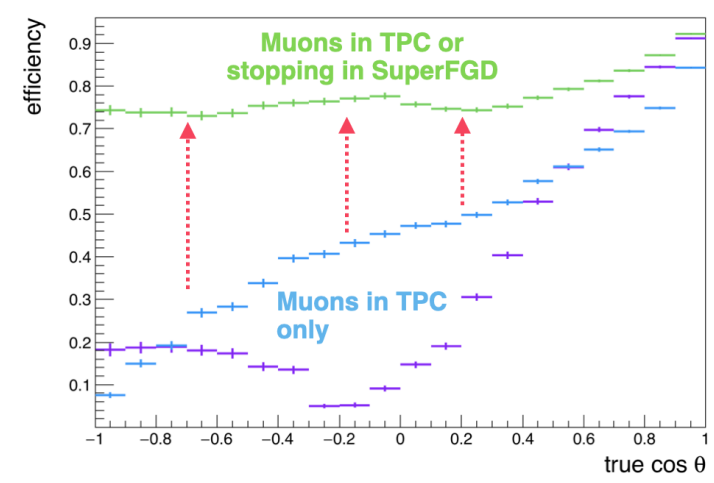

Figure 6: Selection efficiency as a function of the muon angle with respect to the longitudinal axis of CC- $\nu_{\mu}$ events. Purple markers correspond to ND280 current-like. Blue markers correspond to ND280 upgrade-like configuration when the muon is detected by the TPCs, while the green markers also include events where the muons stop inside SuperFGD. The efficiency is shown for events selected in each different target detector.

[2] K. Abe et al. (T2K Collaboration), Phys. Rev. Lett. 107, 041801 (2011), 10.1103/PhysRevLett.107.041801

[3] K. Abe et al. (T2K Collaboration), Phys. Rev. Lett 118, 151801 (2017), 10.1103/PhysRevLett.118.151801

[4] K. Abe et al. (T2K Collaboration), Nature volume 580, pages339-344(2020)

[5] K. Abe et al. (T2K Collaboration), arXiv:1609.04111

[6] K. Abe et al. (T2K Collaboration), arXiv:1901.03750, CERN-SPSC-2018-001 / SPSC-P-357

[7] A. Blondel et al., A fully active fine grained detector with three readout views, JINST $\mathbf{1 3}$ (2018) no.02, P02006 doi:10.1088/1748-0221/13/02/P02006 [arXiv:1707.01785 [physics.ins-det]].

[8] E. Noah et al., Readout scheme for the Baby-MIND detector, PoS PhotoDet 2015 (2016) 031. doi:10.22323/1.252.0031

[9] A. Blondel et al., "The SuperFGD Prototype Charged Particle Beam Tests", arXiv:2008.08861

[10] I. Giomataris, et al., Nucl. Instr. and Meth. A 560 (2006) 405, 10.1016/j.nima.2005.12.222

[11] T. Alexopoulos et al., Nuclear Instruments and Methods in Physics Research A 640, 110 (2011),10.1016/j.nima.2011.03.025

[12] NIMA 518 (2004), p.721

[13] D. Attié et al., Nucl.Instrum.Meth.A 957 (2020) 163286

[14] A. Korzenev et al., arXiv:1901.07785

[15] X.-G. Lu et al., PRC 94,015503 2016

[16] L. Munteanu et. al, PRD 101, 092003 (2020) 